\title{
Perfil de flexibilidad de la extremidad inferior en jugadores senior de balonmano
}

\section{Normative data of Lower-limb muscle flexibility in senior handball players}

\author{
Perfil de flexibilidade de membros inferiores em \\ jogadores de handebol de alto nível
}

\author{
Antonio Cejudo ${ }^{1}$, Pilar Sainz de Baranda ${ }^{1}$, Francisco Ayala ${ }^{2}$ y Fernando Santonja ${ }^{1}$
}

${ }^{1}$ Universidad de Murcia; 2 Universidad Miguel Hernández de Elche.

\begin{abstract}
Resumen: El objetivo de este estudio fue definir los valores de referencia del perfil de flexibilidad de la extremidad inferior en jugadores senior de balonmano. Para ello, se valoró la flexibilidad de los principales grupos musculares de la extremidad inferior (psoas ilíaco, cuádriceps, aductores, isquiosurales, glúteo mayor, gemelo y soleo). 56 jugadores senior de balonmano con más de 9 años de experiencia tomaron parte en este estudio. Los resultados del presente estudio definen como perfil de flexibilidad de la muestra los siguientes valores de referencia: $41,2^{\circ}$ para el gemelo, $43,1^{\circ}$ para el sóleo, $147,8^{\circ}$ para el glúteo mayor, $82,9^{\circ}$ para la musculatura isquiosural, $47,5^{\circ}$ para los aductores, $18,5^{\circ}$ para el psoas iliaco y $125,2^{\circ}$ para el cuádriceps. A su vez, los resultados del presente estudio demuestran que los jugadores de balonmano analizados presentan un perfil de flexibilidad de la extremidad inferior superior a los valores propuestos para población general, a los valores encontrados en sujetos sanos sedentarios, o en personas físicamente activas. De la misma forma, los valores de flexibilidad de los jugadores de balonmano del presente estudio son superiores o similares a los observados en otros deportes (tenis, fútbol, corredores de larga distancia), excepto para la flexibilidad de la musculatura isquiosural y del psoas iliaco. Finalmente, no se han encontrado desequilibrios musculares contra laterales entre ambos lados corporales en ninguno de los músculos evaluados.
\end{abstract}

Palabras clave: Flexibilidad, rango de movimiento, condición física, deportes.

Abstract: The purpose of this study was to define the normative lower-limb flexibility data in senior handball players. For it, the flexibility of the major lower-limb muscles was evaluated throughout 7 different peak joint ROM assessment tests (iliopsoas, quadriceps, adductors, hamstrings, gluteus, gastrocnemius, soleus). 56 senior handball players with more than 9 years of sports experience took part in this study. The results of this study define the normative data as: $41,2^{\circ}$ for the gastrocnemius, $43,1^{\circ}$ for the soleus, $147,8^{\circ}$ for the gluteus, $82,9^{\circ}$ for the hamstrings, $47,5^{\circ}$ for the adductors, $18,5^{\circ}$ for the iliopsoas, and $125,2^{\circ}$ for the quadriceps. The results of the current study demonstrate that the senior handball players assessed have higher normative data of lower-limb flexibility than the previously stabilised flexibility normative data for general population, healthy sedentary adults, as well as for recreationally active adults. Likewise, the normative lower-limb flexibility data for senior handball players are higher or similar than those reported for other sport (tennis, soccer, long distance runners), except for hamstring and iliopsoas flexibility. Finally, no significant differences were found between dominant and non-dominant leg and therefore contra lateral muscle imbalances have been rejected.

Key words: Flexibility, range of motion, physical fitness, sports.

\section{Introducción}

La flexibilidad, definida como la habilidad para mover una articulación (o varias en serie) a través de todo el rango de movimiento $(\mathrm{RdM})$ requerido para una actividad o acción específica (Magnusson y Renstrom, 2006), es uno de los componentes básicos del fitness físico-deportivo (Alricsson y Werner, 2004; Hahn, Foldspang, Vestergaard e IngemannHansen, 1999).

Este hecho, se evidencia con mayor claridad en determinados deportes, tales como la gimnasia rítmica y artística, los saltos en natación y el patinaje artístico, donde la flexibilidad es

Dirección para correspondencia [Correspodence address]: Pilar Sainz de Baranda. Facultad de Ciencias del Deporte. C/Argentina s/n, 30720. Santiago de la Ribera-San Javier (Murcia). E-mail: psainzdebaranda@ um.es un componente determinante del éxito deportivo. Por el contrario, existen otros deportes (fútbol, baloncesto y carrera) que necesitan niveles mas reducidos de flexibilidad para la realización de los movimientos dinámicos implícitos en la ejecución de sus gestos técnicos (Nóbrega, Paula y Carvalho, 2005). Por lo tanto, se podría concluir que la flexibilidad presenta una implicación en mayor o menor medida en cada deporte.

La literatura científica demuestra que dentro de un mismo deporte, la flexibilidad es específica de cada articulación, acción muscular o movimiento (Zakas, Vergou, Zakas, Grammatikopoulou y Grammatikopoulou, 2002). Además, entre los practicantes de un deporte se han encontrado diferentes categorías o niveles de flexibilidad en función del: 1) puesto específico (Oberg, Ekstrand, Möller y Gillquist, 1984); 2) nivel competitivo (élite vs aficionados) (Haff, 2006; Ostojić y Stojanović, 2007); 
así como 3) del segmento corporal (dominante vs no dominante) (Chandler et al., 1990; Probst, Fletcher y Seeling, 2007).

El establecimiento del perfil de flexibilidad en un deporte puede ser una herramienta muy útil para los diferentes profesionales del ámbito de las Ciencias del Deporte, pues permitirá conocer los valores de referencia o normativos que son necesarios alcanzar para: 1) optimizar el rendimiento físicotécnico deportivo, y 2) recuperar el RdM óptimo durante la readaptación física de una lesión deportiva. Además, estos valores de referencia podrían ser utilizados como objetivos específicos cuantificables del entrenamiento de la flexibilidad como cualidad física básica para lograr el éxito deportivo.

Sin embargo, no se han encontrado estudios científicos que hayan determinado el perfil de flexibilidad de jugadores de balonmano. Por ello, el objetivo principal de este estudio fue definir cuantitativamente los valores normativos del perfil de flexibilidad en jugadores senior de balonmano, midiendo para ello la extensibilidad de los principales grupos musculares de la extremidad inferior a través de pruebas angulares pasivas máximas.

\section{Material y Método}

\section{Participantes}

Un total de 50 jugadores de campo y 6 porteros, con más de 9 años de práctica deportiva federada en balonmano (3 sesiones de entrenamiento semanal con una duración mínima de 1,5 horas por sesión), participaron voluntariamente en este estudio. 25 jugadores jugaban en la $1^{\text {a }}$ División Nacional (edad: 26,3 $\pm 6,1$ años; peso: $93 \pm 9,4 \mathrm{Kgs}$; talla: $1,84 \pm 0,07 \mathrm{~m}$ ) y 31 jugadores competía en la 2a División Nacional (edad: 26,2 $\pm 4,1$ ańos; peso: $82,5 \pm 9,3 \mathrm{Kgs}$; talla: $1,78 \pm 0,07 \mathrm{~m}) \mathrm{du}-$ rante la temporada 2010/11.

Fueron excluidos los jugadores que presentaban agujetas en el momento de la valoración o lesiones de la extremidad inferior que pudieran verse agravadas por la realización del estudio y/o desvirtuar los resultados obtenidos.

Tanto los deportistas como los entrenadores fueron verbalmente informados de la metodología a utilizar, así como de los propósitos y posibles riesgos del estudio, y cada uno de ellos firmó un consentimiento informado. El presente estudio fue aprobado por el Comité Ético y Científico de la Universidad de Murcia (España).

\section{Procedimiento}

Una semana antes del inicio del estudio, todos los participantes completaron un cuestionario medico-deportivo (datos personales, datos antropométricos, datos deportivos, historial de lesiones, experiencia con los estiramientos y grupos musculares estresados durante la competición), además de ser sometidos a una sesión de familiarización con el propósito de conocer la correcta ejecución técnica de las pruebas exploratorias mediante la realización práctica de cada una de ellas. Igualmente, otro propósito de esta sesión de familiarización fue la reducción del posible sesgo de aprendizaje sobre los resultados obtenidos en las diferentes pruebas de valoración (Ayala y Sainz de Baranda, 2011). Para conocer la extremidad dominante se pidió a cada jugador realizar tres pruebas: 1) saltar sobre una pierna; 2) golpear una pelota y 3) subirse a un taburete con una pierna, siguiendo la metodología de Wang, Whitney, Burdett y Janosky (1993). La extremidad con la que se ejecutaron al menos 2 de las 3 pruebas fue designado como dominante.

Para el proceso de valoración de la flexibilidad de los principales grupos musculares de la extremidad inferior se siguieron las recomendaciones establecidas por la American Academic of Orthopedic Association (1965) y la American Medical Association [AMA] (Gerhardt, Cocchiarella y Lea, 2002). Los examinadores fueron ciegos en cuanto al objetivo del estudio. Además, los participantes fueron instados a realizar la sesión de valoración en el mismo día y franja horaria que normalmente realizaban sus sesiones de entrenamiento para minimizar la variabilidad intra-sujeto (Hopkins, 2000).

Durante la sesión de valoración, y previo a la aplicación de las diferentes pruebas exploratorias del $\mathrm{RdM}$ pasivo máximo, todos los participantes realizaron 8-10 minutos de calentamiento aeróbico unido a una serie de ejercicios de estiramientos estáticos estandarizados, enfatizando la actividad de los músculos de la extremidad inferior, bajo la estricta supervisión de los examinadores. La intensidad y duración del calentamiento y los ejercicios de estiramiento fueron seleccionados tratando de imitar cada una de las posiciones adoptadas en las pruebas de valoración seleccionadas siguiendo las recomendaciones de Ayala y Sainz de Baranda (2011).

\section{Pruebas de valoración}

Una vez finalizado el calentamiento y los estiramientos, la flexibilidad (expresada en términos cuantitativos en grados) a través del Rango de Movimiento [RdM] pasivo máximo) de los principales grupos musculares de la extremidad inferior (glúteo mayor, psoas ilíaco, aductores, cuádriceps, isquiosurales, gemelo y soleo) se determinó indirectamente a través de 7 pruebas angulares (Figura 1). Los jugadores fueron instados a realizar dos intentos máximos para cada una de las pruebas de valoración y segmento corporal (dominante y no dominante) de forma aleatoria con el propósito de eliminar el sesgo que una secuencia específica podría presentar sobre los resultados obtenidos. La aleatorización en la realización de las pruebas de valoración se llevo a cabo a través del empleo del software informático presente en http://www.randomizer.org. 
Figura 1. Representación gráfica de las 7 pruebas de valoración del rango de movimiento pasivo máximo utilizadas en el presente estudio.

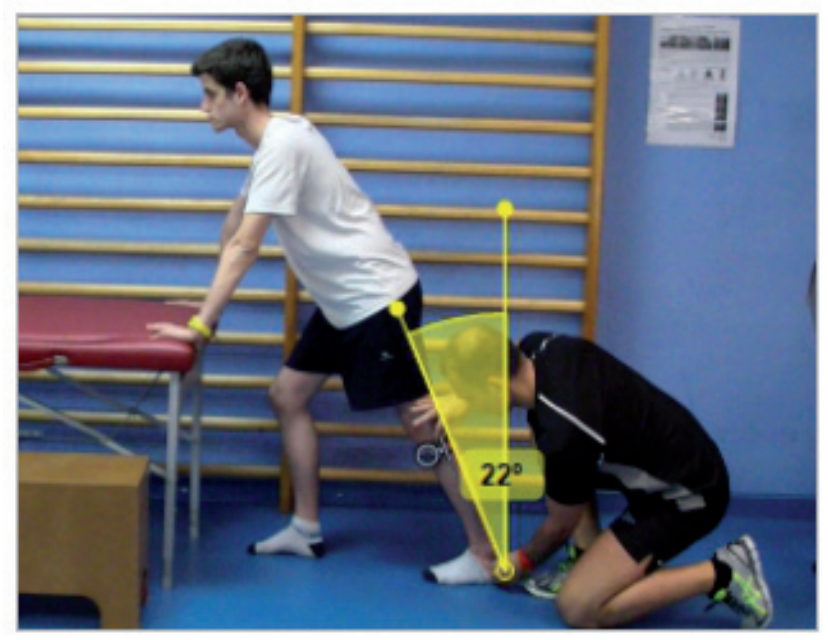

Gemelo

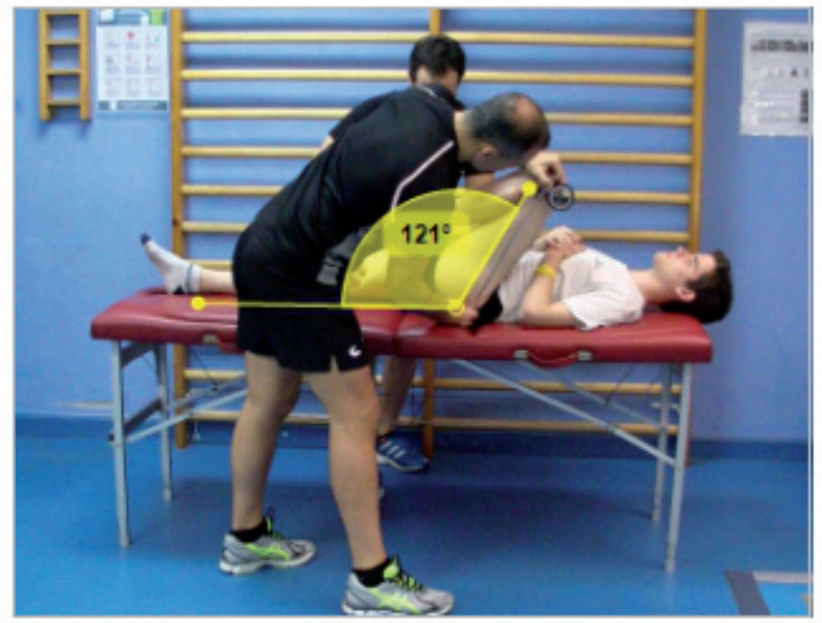

Glúteo mayor

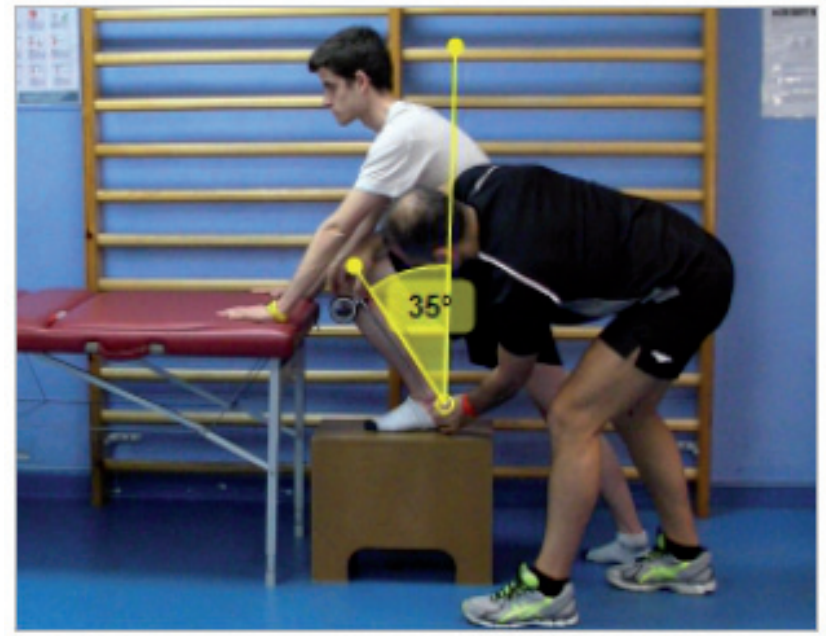

Sóleo

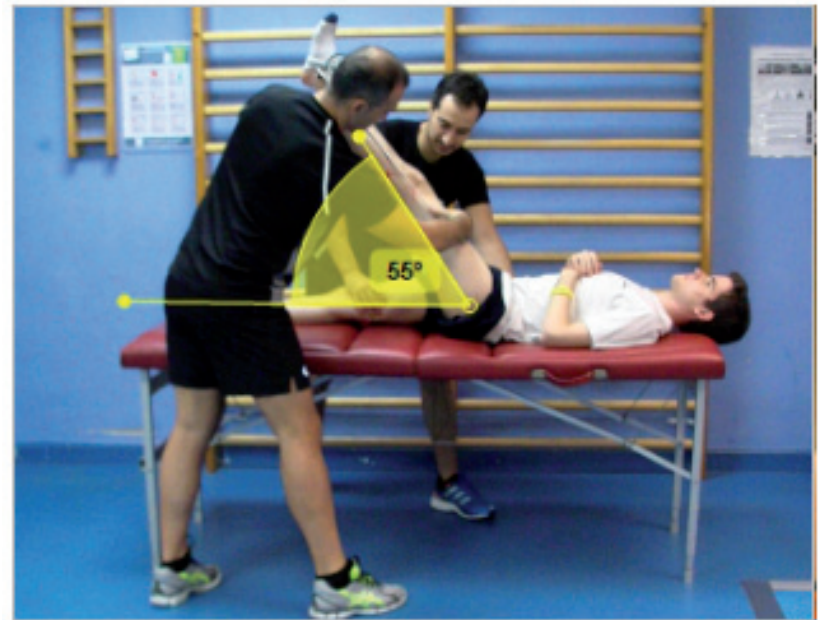

Isquiosural 


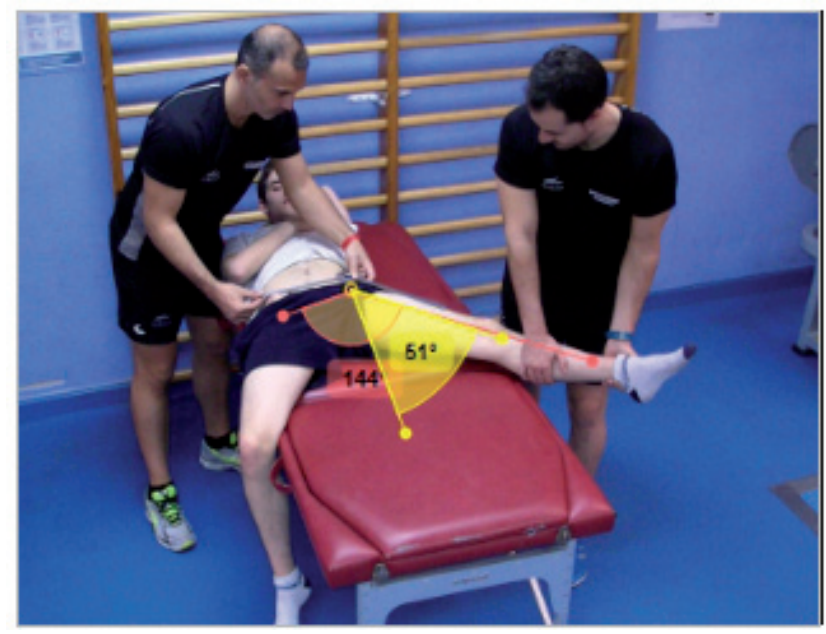

Aductores

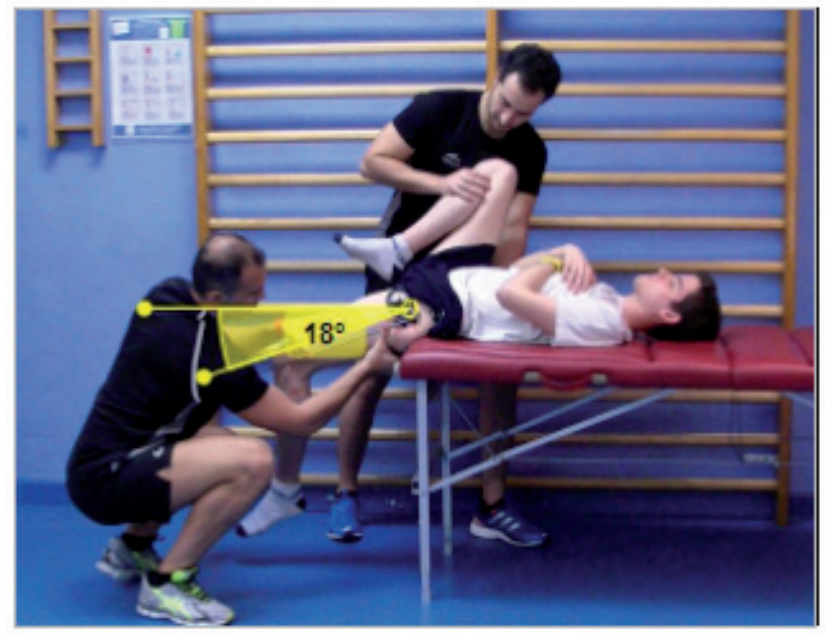

Psoas iliaco

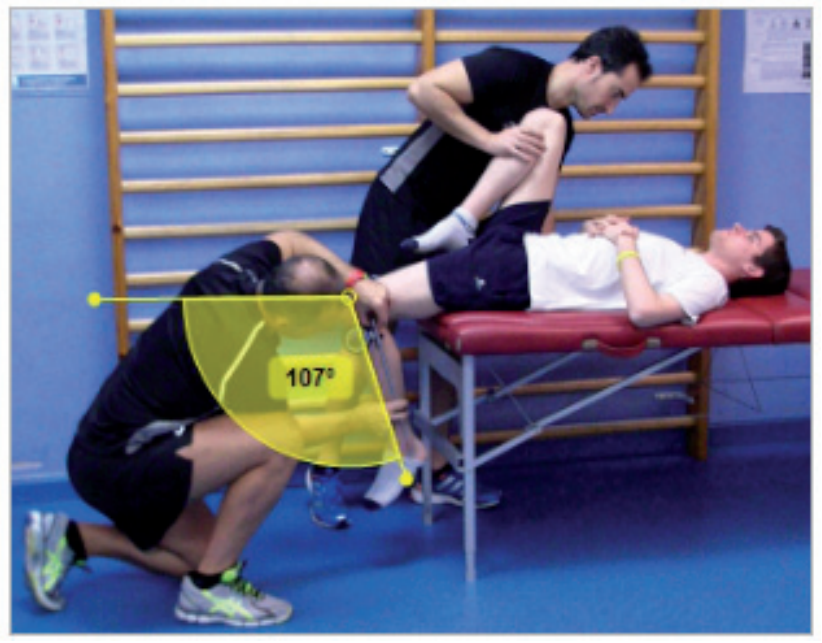

Cuádriceps 
Para la sesión de valoración de la flexibilidad se utilizó una camilla ajustable y como instrumento de medición se utilizó un inclinómetro ISOMED Unilevel con varilla telescópica, siguiendo las recomendaciones de Gerhardt (1994) y Gerhardt et al. (2002).

Cada participante fue valorado con ropa deportiva y sin calzado. Se permitió un periodo de descanso de aproximadamente de 30 segundos entre cada uno de los dos intentos máximos, extremidad y prueba.

El resultado final de cada intento máximo para cada una de las pruebas de valoración fue determinado por uno o varios de los siguientes criterios: 1) el explorador era incapaz de ejecutar de forma lenta y progresiva (sin tirones) el movimiento evaluado debido a la elevada resistencia desarrollada por el/los grupo/s muscular/es estirados (American Academic of Orthopedic Association, 1965); 2) el participante avisaba de sentir tensión o una sensación de estiramiento muscular que acarreaba un disconfort importante (Ekstrand, Wiktorsson, Oberg y Gillquist, 1982; Zakas, Vergou, Grammatikopoulou, Sentelidis y Vamvakoudis, 2003); o 3) ambos exploradores apreciaban algún movimiento de compensación que incrementaba el RdM (Ekstrand et al. 1982; Sainz de Baranda y Ayala, 2010).

El valor medio de cada par de intentos para cada prueba de valoración fue seleccionado para el posterior análisis estadístico (Ayala y Sainz de Baranda, 2011). Sin embargo, cuando una diferencia mayor del 5\% fue observada entre el valor de cada par de intentos para cada prueba exploratoria, un tercer intento fue realizado, seleccionando el valor medio de los dos intentos cuyos resultados estuvieron más próximos para el posterior análisis estadístico. La justificación de la ejecución de 2 intentos máximos para cada prueba de valoración y el cálculo de la media para el posterior análisis estadístico estuvo fundamentada en los resultados de fiabilidad intrasesión obtenidos previamente por los examinadores para cada una de las maniobras exploratorias. Así, un mes antes de la sesión de valoración se realizó un estudio a doble ciego con 20 sujetos físicamente activos (diferentes a los componentes de la muestra del presente estudio), empleando el clásico diseño test-retest. El RdM fue medido dos veces con un intervalo de 15 minutos, no encontrándose diferencias significativas (systematic bias) para cada una de las pruebas de exploración entre ambas mediciones. La fiabilidad intra-sesión de cada una de las variables se determinó a través del coeficiente de correlación intraclase $\left(\mathrm{ICC}_{21}\right)$ empleando el método previamente descrito por Hopkins (2000) Se obtuvo un ICC superior a 0,90 en todas las pruebas de exploración, lo cual demuestra una alta estabilidad de la medida.

\section{Análisis estadístico}

Previo a todo análisis estadístico, la distribución normal de los datos fue comprobada a través de la prueba KolomogorovSmirnov. Se realizó un análisis descriptivo de cada una de las variables cuantitativas, que incluía la media y su correspondiente desviación típica. Además, una prueba t para muestras relacionadas fue empleada para determinar la existencia de diferencias entre los valores de la extremidad dominante y no dominante. Asimismo, se aplicó una prueba t para muestras independientes para observar las posibles diferencias entre niveles competitivos y entre los jugadores de campo y porteros.

El análisis estadístico fue realizado mediante el paquete estadístico SPSS (Statistical Package for Social Sciences, v. 16.0, para Windows; SPSS Inc, Chicago) con un nivel de significación del 95\% $(\mathrm{p}<0,05)$.

\section{Resultados}

En la tabla 1 y 2 se presentan los resultados de la valoración de la flexibilidad de los 7 grupos musculares estudiados en la muestra de jugadores de campo y porteros respectivamente, diferenciado los datos encontrados entre la pierna dominante y no dominante.

Tabla 1. Valores de rango de movimiento pasivo máximo en jugadores de campo $(\mathrm{n}=50)$ de balonmano.

\begin{tabular}{lllll}
\hline & \multicolumn{2}{l}{$1^{\text {a }}$ División Nacional $(\mathrm{n}=22)$} & \multicolumn{2}{l}{$2^{\text {a }}$ División Nacional $(\mathrm{n}=28)$} \\
\cline { 2 - 5 } & Dominante & No dominante & Dominante & No dominante \\
\hline Gemelo & $40,5^{\circ} \pm 6,1^{\circ}$ & $39,5^{\circ} \pm 5,1^{\circ}$ & $42,0^{\circ} \pm 5,1^{\circ}$ & $42,69^{\circ} \pm 6,5^{\circ}$ \\
Sóleo & $42,4^{\circ} \pm 4,8^{\circ}$ & $42,0^{\circ} \pm 5,4^{\circ}$ & $44,0^{\circ} \pm 6,0^{\circ}$ & $43,45^{\circ} \pm 7,2^{\circ}$ \\
Glúteo mayor & $145,8^{\circ} \pm 4,7^{\circ}$ & $145,5^{\circ} \pm 5,3^{\circ}$ & $149,5^{\circ} \pm 6,9^{\circ}$ & $149,76^{\circ} \pm 6,1^{\circ}$ \\
Isquiosurales & $80,7^{\circ} \pm 12,4^{\circ}$ & $78,3^{\circ} \pm 12,5^{\circ}$ & $86,1^{\circ} \pm 10,1^{\circ}$ & $86,69^{\circ} \pm 9,3^{\circ}$ \\
Aductores & $47,0^{\circ} \pm 7,5^{\circ}$ & $45,0^{\circ} \pm 6,3^{\circ}$ & $48,8^{\circ} \pm 7,9^{\circ}$ & $49,14^{\circ} \pm 7,6^{\circ}$ \\
Psoas-Iliaco & $17,1^{\circ} \pm 5,1^{\circ}$ & $18,6^{\circ} \pm 4,3^{\circ}$ & $18,6^{\circ} \pm 5,8^{\circ}$ & $19,62^{\circ} \pm 6,3^{\circ}$ \\
Cuádriceps & $123,2^{\circ} \pm 10,9^{\circ}$ & $123,8^{\circ} \pm 12,1^{\circ}$ & $126,2^{\circ} \pm 12,7^{\circ}$ & $127,54^{\circ} \pm 11,9^{\circ}$ \\
\hline
\end{tabular}


Tabla 2. Valores de rango de movimiento pasivo máximo en porteros $(\mathrm{n}=6)$ de balonmano.

\begin{tabular}{lllll}
\hline & \multicolumn{2}{l}{$1^{\text {a }}$ División Nacional $(\mathrm{n}=3)$} & \multicolumn{2}{l}{$2^{\text {a }}$ División Nacional $(\mathrm{n}=3)$} \\
\cline { 2 - 5 } & \multicolumn{1}{l}{ Dominante } & No dominante & Dominante & No dominante \\
\hline Gemelo & $47,3^{\circ} \pm 2,3^{\circ}$ & $49^{\circ} \pm 1,4^{\circ}$ & $45^{\circ} \pm 2,2^{\circ}$ & $51^{\circ} \pm 1,41^{\circ}$ \\
Sóleo & $42,3^{\circ} \pm 1,4^{\circ}$ & $45,6^{\circ} \pm 3,7^{\circ}$ & $40^{\circ} \pm 1,5^{\circ}$ & $40,6^{\circ} \pm 2,7^{\circ}$ \\
Glúteo mayor & $151,1^{\circ} \pm 1,1^{\circ}$ & $149,3^{\circ} \pm 4,2^{\circ}$ & $148,6^{\circ} \pm 1,3^{\circ}$ & $146,4^{\circ} \pm 2,2^{\circ}$ \\
Isquiosurales & $107,8^{\circ} \pm 4,4^{\circ *}$ & $104,6^{\circ} \pm 7^{*}$ & $80,8^{\circ} \pm 3,5^{\circ}$ & $80,3^{\circ} \pm 4^{\circ}$ \\
Aductores & $56,5^{\circ} \pm 0,2^{\circ *}$ & $51^{\circ} \pm 1,17^{\circ}$ & $51,5^{\circ} \pm 0,2^{\circ}$ & $50^{\circ} \pm 2,17^{\circ}$ \\
Psoas Iliaco & $20,3^{\circ} \pm 1,8^{\circ}$ & $20,6^{\circ} \pm 0,4^{\circ}$ & $19,2^{\circ} \pm 1,4^{\circ}$ & $22,3^{\circ} \pm 1,3^{\circ}$ \\
Cuádriceps & $137^{\circ} \pm 10,8^{\circ}$ & $132^{\circ} \pm 12,2^{\circ}$ & $137^{\circ} \pm 8,8^{\circ}$ & $133^{\circ} \pm 11,4^{\circ}$ \\
\hline
\end{tabular}

* diferencias significativas entre categorías competitivas $(p<0,05)$.

${ }^{\dagger}$ diferencias significativas entre puestos específicos $(\mathrm{p}<0,05)$.

Cuando se analizaron las diferencias entre ambos niveles competitivos (1ª División Nacional vs 2a División Nacional), solo se encontraron diferencias significativas en los porteros en la flexibilidad aductora del lado dominante $(\mathrm{p}<0,05)$ y en la flexibilidad isquiosural $(\mathrm{p}<0,05)$.

Cuando se analizaron las diferencias entre puestos específicos (jugadores de campo vs porteros) no se encontraron diferencias significativas $(\mathrm{p}>0,05)$ en la categoría de $2^{\text {a }}$ División Nacional. Mientras que en $1^{\text {a }}$ División Nacional se encontraron diferencias significativas en la flexibilidad isquiosural en ambos lados corporales y en la flexibilidad aductora del lado no dominante.

Cuando se analizaron las diferencias entre ambos lados corporales (dominante vs no dominante) mediante la prueba $\mathrm{t}$ student para muestras relacionadas, no se encontraron diferencias estadísticamente significativas en ninguna prueba valorada.

\section{Discusión}

El principal propósito de este trabajo fue definir el perfil de flexibilidad de los principales grupos musculares de la extremidad inferior en una muestra de jugadores senior de balonmano.

Hay que tener en cuenta que la mayoría de los trabajos consultados que valoran la flexibilidad en población general o en otros deportes miden exclusivamente la flexibilidad de uno o dos músculos y/o utilizan pruebas lineales. Como se puede observar en la tabla 3, los valores de flexibilidad del jugador de balonmano son superiores a los valores propuestos para población general (Gerhardt et al., 2002; Palmer y Epler, 2002), a los valores encontrados en sujetos sanos sedentarios (Ekstrand et al., 1982; Clapis, Davis y Davis, 2008) o en personas recreacionalmente activas (Mahieu et al., 2007; Probst et al., 2007).

Tabla 3. Valores de referencia de rango de movimiento en goniometría y en población adulta no deportista.

\begin{tabular}{|c|c|c|c|c|c|c|c|}
\hline & Gemelo & Sóleo & Glúteo & Isquios & Aductores & Psoas & Cuádriceps \\
\hline $\begin{array}{l}\text { Balonmano } \\
\text { Presente estudio }\end{array}$ & $41,2^{\circ}$ & $43,1^{\circ}$ & $147,8^{\circ}$ & $82,9^{\circ}$ & $47,5^{\circ}$ & $18,5^{\circ}$ & $125,2^{\circ}$ \\
\hline Goniometría $^{1}$ & $0^{\circ}-30^{\circ}$ & $0^{\circ}-30^{\circ}$ & $135^{\circ}-140^{\circ}$ & $80^{\circ}$ & $\begin{array}{l}0^{o}-45^{\circ} \\
0^{\circ}-50^{o \ddagger}\end{array}$ & $0^{\circ}-30^{\circ}$ & $0^{\circ}-120^{\circ} / 125^{\circ}$ \\
\hline \multicolumn{8}{|l|}{$\begin{array}{l}\text { Población adulta } \\
\text { no deportista }\end{array}$} \\
\hline Ekstrand et al. (1982) & $22,5^{\circ}$ & $24,9^{\circ}$ & & $81^{\circ}$ & $37^{\circ}$ & $6,5^{\circ}$ & \\
\hline Clapis et al. (2008) & & & & & & $\begin{array}{l}-1,7^{\mathrm{o}^{\dagger}} \\
-2,8^{\mathrm{o}^{\ddagger}}\end{array}$ & \\
\hline Mahieu et al. (2007) & $28,4^{\circ}$ & $36,7^{\circ}$ & & & & & \\
\hline Probst et al. (2007) & & & & & & $20,4^{\mathrm{o}}$ & \\
\hline
\end{tabular}


De la misma forma, los valores de flexibilidad de los jugadores de balonmano del presente estudio son superiores o similares a los observados en otros deportes (tenis, fútbol, corredores de larga distancia), excepto para la flexibilidad isquiosural y del psoas iliaco.

Con relación al balonmano se han encontrado 3 trabajos que han valorado la flexibilidad en jugadores junior (Gruić, Ohnjec y Vuleta, 2011; Zakas et al., 2002; Zakas et al., 2003) y senior profesionales (Gruić et al., 2011). De forma general, los jugadores del presente estudio muestran mejores o similares valores en la flexibilidad del soleo, aductores y psoas iliaco. Mientras que para la flexibilidad isquiosural los jugadores del presente estudio muestran mejores o similares valores que los jugadores de balonmano junior y menores valores que los jugadores de balonmano senior profesionales de nivel internacional (Gruić et al., 2011) (Tabla 4). Este hecho, puede justificarse por el mayor nivel competitivo de la muestra valorada por Gruić et al. (2011), ya que ha sido demostrado en otros trabajos, que los deportistas de mayor nivel competitivo presentan mejores valores de flexibilidad (Gruić et al., 2011; Haff, 2006; Ostojić y Stojanović, 2007). Aunque en el presente trabajo tan solo se hayan observado diferencias estadísticamente significativas entre ambos niveles competitivos (1a División Nacional vs $2^{\text {a }}$ División Nacional) en los porteros para la flexibilidad de los aductores de la extremidad dominante y para la flexibilidad isquiosural.

Tabla 4. Valores de rango de movimiento en diferentes deportes empleando las mismas pruebas descritas en el presente estudio.

\begin{tabular}{|c|c|c|c|c|c|c|c|}
\hline & Gemelo & Sóleo & Glúteo M. & Isquios & Aductores & Psoas & Cuádriceps \\
\hline $\begin{array}{l}\text { Balonmano } \\
\text { Presente estudio }\end{array}$ & $41,2^{\circ}$ & $43,1^{\circ}$ & $147,8^{\circ}$ & $82,9^{\circ}$ & $47,5^{\circ}$ & $18,5^{\circ}$ & $125,2^{\circ}$ \\
\hline $\begin{array}{l}\text { Balonmano junior } \\
\text { (Zakas et al., 2002) }\end{array}$ & & $29,8^{\circ}$ & & $76,3^{\circ}$ & $46,5^{\circ}$ & $8,2^{\circ}$ & \\
\hline $\begin{array}{l}\text { Balonmano junior } \\
\text { (Zakas et al., 2003) }\end{array}$ & & $29,2^{\circ}$ & & $79,8^{\circ}$ & $45,3^{\circ}$ & $8,2^{\circ}$ & \\
\hline $\begin{array}{l}\text { Balonmano junior profesional } \\
\text { (Gruić et al., 2011) }\end{array}$ & & & & $\begin{array}{l}82,8^{\mathrm{o}^{\dagger}} \\
85,9^{\mathrm{o}^{\ddagger}}\end{array}$ & & & \\
\hline $\begin{array}{l}\text { Balonmano senior profesional } \\
\text { (Gruić et al., 2011) }\end{array}$ & & & & $\begin{array}{l}93,8^{\mathrm{o}^{\dagger}} \\
95,2^{\mathrm{o}}\end{array}$ & & & \\
\hline $\begin{array}{l}\text { Fútbol masculino } \\
\text { (Ekstrand et al., 1982) }\end{array}$ & $21,4^{\circ}$ & & $102^{\circ}$ & $80,8^{\circ}$ & $33,5^{\circ}$ & $9,1^{\circ}$ & \\
\hline $\begin{array}{l}\text { Tenis elite junior masculino } \\
\text { (Chandler et al., 1990) }\end{array}$ & & & & $77,5^{\circ}$ & & & $124^{\circ}$ \\
\hline $\begin{array}{l}\text { Tenis elite junior masculino } \\
\text { (Kibler et al., 2003) }\end{array}$ & & & & $65,9^{\circ}$ & & & $123,1^{\circ}$ \\
\hline $\begin{array}{l}\text { Esquiadores de fondo } \\
\text { (Alricsson y Werner, 2004) }\end{array}$ & $35,5^{\circ}$ & & & $99^{\circ}$ & & & \\
\hline $\begin{array}{l}\text { Corredores } \\
\text { a) } 30-55 \text { millas/semana } \\
\text { b) } 76 \text { millas/semana } \\
\text { (Wang et al., 1993) }\end{array}$ & $\begin{array}{l}\text { a) } 9,1^{\circ} \\
\text { b) } 6,6^{\circ}\end{array}$ & $\begin{array}{l}\text { a) } 19,1^{\circ} \\
\text { b) } 16,3^{\circ}\end{array}$ & & $\begin{array}{l}\text { a) } 76,7^{\circ} \\
\text { b) } 65,5^{\circ}\end{array}$ & & & \\
\hline Karate (Probst et al., 2007) & & & & & & $22,4^{\circ}$ & \\
\hline
\end{tabular}

Por otro lado, la literatura científica informa de que el nivel de flexibilidad es específico en función del puesto que ocupe cada jugador en un mismo deporte (Oberg et al., 1984), y generalmente, se acepta que los porteros presentan mayores valores que los jugadores de campo (Arnason, Sigurdsson, Gudmundsson, Holme, Engebretsen y Bahr, 2004; Gleim, 1984; Oberg et al., 1984). Los resultados del presente trabajo no coinciden en parte con esta idea, ya que en la mayoría de las pruebas no se han encontrado diferencias significativas entre puestos específicos. Así, sólo se han encontrado diferencias significativas en la flexibilidad isquiosural y la aductora del lado no dominante, a favor de los porteros.

Además, y como parte del análisis del perfil de flexibilidad, la literatura científica propone valorar las diferencias entre el tipo de la extremidad dominante o no dominante (Chandler et al., 1990; Probst et al., 2007), ya que la repetición de los gestos técnicos y las exigencias físicas específicas pueden ocasionar adaptaciones músculo-esqueléticas negativas como 
la cortedad muscular (Kibler y Chandler, 2003; Wang et al., 1993).

Los desequilibrios de flexibilidad entre el lado dominante $y$ no dominante se ha observado en el hombro en tenistas (Chandler, Kibler, Stracener, Ziegler y Pace, 1992), en la posición de pitcher en béisbol (Hinton, 1988), en nadadores (Weldon y Richardson, 2001) y en jugadores de voleibol (Cools, Witvrouw, Mahieu y Danneels, 2005), y han sido relacionados con un incremento del riesgo de lesión deportiva (Bozic, Pazin, Berjan, Planic y Cuk, 2010; Probst et al., 2007). Por ello, en la valoración de la flexibilidad del presente estudio se ha definido el perfil de flexibilidad para el lado dominante y no dominante y en función del puesto específico. El análisis estadístico no ha mostrado diferencias significativas entre ambos lados corporales en la extensibilidad de los músculos valorados, por lo que los jugadores de estos equipos presentan un menor probabilidad de riesgo de lesión.

\section{Aplicaciones prácticas}

El establecimiento del perfil de flexibilidad es una herramienta básica que debe incluirse en cualquier protocolo que valore la condición física del deportista. Las razones que justifican su inclusión y análisis son: 1) Permite conocer los valores de referencia o normativos que son necesarios para alcanzar y lograr el éxito deportivo; 2) Permite identificar adaptaciones negativas del deporte como la cortedad muscular, las asimetrías musculares o estimar cierto riesgo de lesión; 3) Los valores de referencia pueden ser utilizados como objetivos específicos cuantificables del entrenamiento de la flexibilidad o como valores a recuperar durante el proceso de readaptación física en una lesión deportiva.

\section{Conclusión}

Los resultados del presente estudio definen como perfil de flexibilidad de los jugadores senior de balonmano evaluados los siguientes valores de referencia: $41,2^{\circ}$ para el gemelo, $43,1^{\circ}$ para el sóleo, $147,8^{\circ}$ para el glúteo mayor, $82,9^{\circ}$ para la musculatura isquiosural, $47,5^{\circ}$ para los aductores, $18,5^{\circ}$ para el psoas-iliaco y $125,2^{\circ}$ para el cuádriceps. Los jugadores de balonmano analizados presentan un perfil de flexibilidad superior a los valores propuestos para población general, a los valores encontrados en sujetos sanos sedentarios, o en personas físicamente activas. De la misma forma, los valores de flexibilidad son superiores o similares a los observados en otros deportes (tenis, fútbol, corredores de larga distancia), excepto para la musculatura isquiosural y el psoas iliaco. Finalmente, no se han encontrado diferencias contralaterales de flexibilidad entre la extremidad dominante y no dominante en los músculos evaluados.

\section{Referencias Bibliográficas}

1. Alricsson, M. y Werner, S. (2004). The effect of pre-season dance training on physical indices and back pain in elite cross-country skiers: a prospective controlled intervention study. British Journal of Sports $\mathrm{Me}$ dicine, 38(2), 148-153.

2. American Academy of Orthopaedic Association. (1965). Joint Motion: Method of Measuring and Recording. Chicago: Park Ridge.

3. Arnason, A., Sigurdsson, S.B., Gudmundsson, A., Holme, I., Engebretsen, L. y Bahr, R. (2004). Physical fitness, injuries, and the team performance in soccer. Medicine and science in sports and exercise, 36(2), 278-285.

4. Ayala, F. y Sainz de Baranda, P. (2011). Reproducibilidad inter-sesión de las pruebas distancia dedos planta y distancia dedos suelo para estimar la flexibilidad isquiosural en jugadores adultos de fútbol sala de primera división. Revista Andaluza de Medicina del Deporte, 4(2), 47-51.

5. Bozic, P.R., Pazin, N.R., Berjan, B.B., Planic, N.M. y Cuk, I.D. (2010) Evaluation of the field tests of flexibility of the lower extremity: reliability and the concurrent and factorial validity. Journal of Strength and Conditioning Research, 24(9), 2523-2531.

6. Chandler, T.J., Kibler, W.B., Stracener, E.C., Ziegler, A.K. y Pace, B. (1992). Shoulder strength, power and endurance in college tennis players. American Journal of Sports Medicine, 20(4), 455-458.

7. Chandler, T.J., Kibler, W.B., Uhl, T.L., Wooten, B., Kiser, A. y Stone, E. (1990). Flexibility comparisons of the junior elite tennis players to other athlete. American Journal of Sports Medicine, 18(2), 134-136.

8. Clapis, P.A., Davis, S.M. y Davis, R.O. (2008). Reliability of inclinometer and goniometric measurements of hip extension flexibility using the modified Thomas test. Physiotherapy Theory and Practice, 24(2), 135141.
9. Clarkson, H.M. (2003). Proceso evaluativo músculoesquelético. Barcelona: Paidotribo.

10. Cools, A.M., Witvrouw, E.E., Mahieu, N.N. y Danneels, L.A. (2005). Isokinetic scapular muscle performance in overhead athletes with and without impingement symptoms. Journal of Athletic Training, 40(2), 104-110.

11. Ekstrand, J., Wiktorsson, M., Oberg, B. y Gillquist, J. (1982). Lower extremity goniometric measurements: A study to determine their reliability. Archives of Physical Medicine and Rehabilitation, 63(4), 171-175.

12. Gerhardt, J. (1994). Documentation of Joint Motion. Oregon: Isomed.

13. Gerhardt, J., Cocchiarella, L. y Lea, R. (2002). The Practical Guide to Range of Motion Assessment. Chicago: American Medical Association.

14. Gleim, G.W. (1984). The profiling of professional football players. Clinical Journal of Sport Medicine, 3,185-197.

15. Gruić, I., Ohnjec, K. y Vuleta, D. (2011). Comparison and analyses of differences in flexibility among top-level male and female handball players of different ages. FACTA UNIVERSITATIS Series: Physical Education and Sport, 9(1), 1-7.

16. Haff, G. (2006). Roundtable Discussion: Flexibility training. Strength and Conditioning Journal, 28(2), 64-85.

17. Hahn, T., Foldspang, A., Vestergaard, E. y Ingemann-Hansen, T. (1999). Active knee joint flexibility and sports activity. Scandinavian Journal of Medicine \& Science in Sports, 9(2), 74-80.

18. Hinton, R.Y. (1988). Isokinetic evaluation of shoulder strength in high school baseball pitchers. American Journal of Sports Science and Medicine, 16(3), 274-279.

19. Hopkins, W.G. (2000). Measures of reliability in sports medicine and science. Sports Medicine, 30, 1-15. 
20. Kibler, W.B. y Chandler, T.J. (2003). Range of movement in junior tennis player participating in an injury risk modification program. Journal of Science and Medicine in Sport, 6(1), 51-62.

21. Magnusson, P. y Renström, P. (2006). The European College of Sports Sciences Position statement: The role of stretching exercises in sports. European Journal of Sport Science, 6(2), 87-91.

22. Mahieu, N.N., McNair, P., De Muynck, M., Stevens, V., Blanckaert, I., Smits, N. y Witvrouw, E. (2007). Effect of static and ballistic stretching on the muscle-tendon tissue properties. Medicine and science in sports and exercise, 39(3), 494-501.

23. Nóbrega, A.C., Paula, K.C. y Carvalho, A.C. (2005). Interaction between resistance training and flexibility training in healthy young adults. Journal of Strength and Conditioning Research, 19(4), 842-846.

24. Norkin, C. y White, J. (2006). Goniometría. Evaluación de la Movididad Articular. Madrid, Marban.

25. Oberg, B.; Ekstrand, J.; Möller, M. \& Gillquist, J. (1984). Muscle strength and flexibility in different positions of soccer players. International Journal of Sports Medicine, 5(4), 213-216.

26. Ostojić, S.M. y Stojanović, M.D. (2007). Range of motion in the lower extremity: elite vs. non-elite soccer players. Serbian Journal of Sports Sciences, 1(2), 74-78.

27. Palmer, M.L. y Epler, M.E. (2002). Fundamentos de las técnicas de la evaluación musculoesquelética. Barcelona: Paidotribo.

28. Probst, M.M., Fletcher, R. y Seeling, D.S. (2007). A comparison of lower-boy flexibility strength, and knee stability between karate athletes and active controls. Journal of Strength and Conditioning Research, 21(2), 451-455.

29. Sady, S.P., Wortman, M. y Blanke, D. (1982). Flexibility training: ballistic, static or proprioceptive neuromuscular facilitation?. Archives of Physical Medicine and Rehabilitation, 63(6), 261-263.

30. Sainz de Baranda, P. y Ayala, F. (2010). Chronic flexibility improvement after 12 week stretching program utilizing the ACSM recommendations: Hamstring flexibility. International Journal of Sports Medicine, 31(6), 389-396.

31. Wang, S.S., Whitney, S.L., Burdett, R.G. y Janosky J.E. (1993). Lower extremity muscular flexibility in long distance runners. Journal of Orthopaedic and Sports Physical Therapy, 17(2), 102-107.

32. Weldon, E.J.3 ${ }^{\text {rd }}$ y Richardson, A.B. (2001). Upper extremity overuse injuries in swimming. A discussion of swimmer's shoulder. Clinical Journal of Sport Medicine, 20(3), 423-438.

33. Zakas, A., Vergou, A., Zakas, N., Grammatikopoulou, M.G. y Grammatikopoulou, G.T. (2002). Handball match effect on the flexibility of junior handball players. Journal of Human Movement Studies, 43, 321330.

34. Zakas, A., Vergou, M., Grammatikopoulou, N., Sentelidis, T. y Vamvakoudis, S. (2003). The effect of stretching during warming up on the flexibility of junior handball players. The Journal of Sports Medicine and Physical Fitness, 43(2), 145-149. 
